\title{
TTR
}

Traduction, terminologie, re?daction

\section{Anthony Pym. Pour une éthique du traducteur. Arras, Artois Presses Université, Presses de l'Université d'Ottawa, 1997.}

\section{Alexis Nouss}

Volume 11, numéro 1, 1er semestre 1998

Diachronie et synchronie

Diachronics and Synchronics

URI : https://id.erudit.org/iderudit/037327ar

DOI : https://doi.org/10.7202/037327ar

Aller au sommaire du numéro

\section{Éditeur(s)}

Association canadienne de traductologie

ISSN

0835-8443 (imprimé)

1708-2188 (numérique)

Découvrir la revue

Citer ce compte rendu

Nouss, A. (1998). Compte rendu de [Anthony Pym. Pour une éthique du traducteur. Arras, Artois Presses Université, Presses de l'Université d'Ottawa, 1997.] TTR, 11(1), 258-263. https://doi.org/10.7202/037327ar

Tous droits réservés @ C TTR: traduction, terminologie, rédaction — Les auteurs, 1998 


\section{Anthony Pym. Pour une éthique du traducteur. Arras, Artois Presses Université, Presses de l'Université d'Ottawa, 1997.}

Titre ambitieux et séduisant. Serait-ce le volume tant attendu en traductologie depuis que la question éthique a été mise de l'avant, dans Ie sillage inaugural de Walter Benjamin puis grâce aux travaux, entre autres, de J. Derrida, H. Meschonnic (étrangement absent dans les références), A. Berman? Par ailleurs, en élargissant notre horizon de lecture, on assiste 
depuis les dernières années à un retour en force de l'ethique - bioéthique, droits d'ingérence, action humanitaire, réfugiés et sans papiers... comme venue pallier un grand vide provoqué par la fin des idéologies. Sur ce point, la traduction, forme exemplaire de dialogue, offre incontestablement des perspectives spécifiques. De surcroît, l'auteur a toujours affiché des positions originales, parfois provocatrices, servies par une interdisciplinarité et un militantisme multiculturel affirmés (voir son Translation and Text Transfer : An Essay on the Principles of Intercultural Communication, Peter Lang, 1992).

Force nous est de contaster que notre attente n'a pas été comblée. Car évoquant l'éthique du traducteur, on est en droit de poser aussi et prioritairement une éthique du traductologue, qui relèverait simplement de l'éthique de toute pensée critique ou métadiscursive, et le présent volume en semble passablement dépourvu. Il apparait davantage comme un plaidoyer pro modo riche de développements fort intéressants mais ne répondant pas à la question qu'il annonce. D'abord eût-il fallu la définir. L'introduction annonçait fort pertinemment la nécessité de substituer la question « Faut-il traduire? * au banal * Comment traduire? n et promettait d'y répondre au nom de "l'espace interculturel du traducteur ", " une intersection entre les cultures n (p. 14), rappelant les noms des traducteurs victimes, dans le passé ou à notre époque, de l'intolérance et du fanatisme. Les chapitres finaux, cependant, ne reprendront la question et n'y répondront qu'en termes de coût et d'investissement. Une attaque réglée contre Berman dont l'éthique est jugée * trop académique, trop intellectuelle, trop abstraite " ne suffit pas non plus. En quoi consiste son échec selon l'auteur? Refusant d'être une éthique professionnelle, elle est restée marginale. En revanche, "[...] nous parlerons, prévient A. Pym dans l'introduction, plutôt de la traduction professionnelle, celle qui se paie, qui s'échange contre de l'argent ou contre une valeur morale. Sous quelque forme que ce soit, la traduction, en principe, se paie. Piste importante dans la pensée éthique " (p. 11). Singulière piste qui associe éthique et intérêt, et la seule, en vérité, dans cette définition restreinte, que suivra l'auteur ne précisant nulle part son rapport à l'éthique en général. Sauf à la page 50 où indiquant brièvement trois finalités de l'éthique dans la pensée occidentale, il s'arrête à Kant. Mais si le discours sur l'éthique est " de retour n aujourd' hui, c'est dans des considérations radicalement renouvelées dont peuvent témoigner, entre autres, les noms de Riccur, Levinas, Jonas, Habermas, Vattimo, Rorty, Taylor, Rawls, Badiou, toutes 
pensées dont l'apport est précisément de théoriser la possibilité d'un discours sur l'éthique soumis à l'histoire, et non d'un discours éthique principiel. La systématicité et la légitimité d'une quelconque transcendance prescriptive sont abandonnées devant la nécessité de s'appuyer sur l'impératif de l'intersubjectivité. Le monde avant tout comme monde humain et relationnel, paysage où la traduction n'aurait aucun mal à se faire accueillir, au contraire.

Mais ces références présentent sans doute quelque chose de suspect pour l'auteur qui n'a de cesse de se démarquer des théoriciens purs, du $\propto$ discours académique * (p. 17), surtout de la * gauche universitaire " (p. 96) - L. Venuti au premier rang -, pour se placer sur le plan de la pratique, dans une stratégie bien connue en traductologie, reconduisant un des binarismes que par ailleurs il dénonce (pp. 21-22). Pourtant, le volume est présenté comme un séminaire donné au College International de Philosophie, à Paris, en 1994 : cautionnement ou, selon un terme qui lui est cher, négociation? Pourtant, ses deux premiers chapitres sont bien théoriques qui s'appuient sur l'Antiquité et les philosophes pour énoncer les thèses de l'auteur sur l'interculturel. Pourtant, il n'hésite pas par la suite à faire appel à Aristote et à ses catégories de causalité (p. 85) pour éclairer la notion de contexte et fonder sa propre typologie des responsabilités (notion qu'il dégage, en une étrange construction éditoriale, au chapitre précédent). A cet égard, l'invitation au lecteur, sous forme d'avertissement (p. 16), de pouvoir lire les six chapitres séparément ne dédouane pas la faiblesse structurale de l'ouvrage : les deux premiers chapitres tentant de cerner dans une approche philosophique l'identité du traducteur et les quatre suivants offrant des considérations pragmatiquement fondées sur une éthique professionnellement orientée, sans que le lien entre les deux parties n'apparaisse évident.

L'analyse (chapitre IV) des theories traductologiques existantes dans l'optique du privilège accordé à la source, à la finalité, à la forme ou au traducteur - quatre modes de causalité - est d'une grande vertu heuristique, de même que l'examen (chapitre III) des aspects de la responsabilité du traducteur. Mais le lien entre causalité et responsabilité, compte tenu de la charge sémantique de ces notions, demeure ténu ou du moins trop dépendant des postulats de l'auteur. * Plus j'investis d'effort dans une traduction, plus je vends mon travail à prix élevé. [...] Bref, la 
question n'est pas seulement de savoir s'il faut traduire ou non, mais de cerner le degré d'effort à investir dans la traduction. Ceci pourrait être une question éthique. " (p. 105) " La seule pensée éthique doit chercher les aspects positifs du commerce, tout en écartant les aspects négatifs. " (p. 132) L'intérêt ne fait pas conceptuellement bon ménage avec la morale. Oublions donc ce que la philosophie nous a préparés à attendre et soyons plutôt attentifs à ce que l'auteur cherche à communiquer.

Son insistance à situer sa problématique dans le cadre sociologique, matériel et financier de la profession et à considérer ce qu'il appelle la " traduction traductionnelle " (p. 75), le produit-traduction, amène à penser que, plutôt qu'éthique, déontologie serait un terme plus approprié pour désigner ce dont traite l'ouvrage. Sur ce plan, le travail d'A. Pym - dont témoignent le présent volume ainsi que ses nombreuses activités dans le milieu traductologique - s'avère un remarquable et sincère combat pour la réhabilitation et la dignité du traducteur. Par ailleurs, à lire les analyses minutieuses consacrées aux rapports entre le traducteur et son " client », on serait porté à croire que le principe majeur de cette déontologie n'est autre que, de part et d'autre, un principe de rentabilite maximale. Lorsque dans une note de la page 111, l'auteur évoque le libéralisme dans le cadre des relations internationales, n'est-ce pas le modèle économique plus que politique auquel il se réfêre? $\mathrm{Si}$ le lecteur accepte une utilisation du concept d'éthique ainsi comprise et rétablit le cadre épistémologique de l'ouvrage, celui-ci livrera des analyses fines et précieuses sur l'exercice de la profession. Les développements d'A. Pym autour des deux notions centrales qu'il dégage, la négociation et la coopération, sont finement argumentés et clairement menés, sis sans un cadre économico-communicationnel. Chiffres et graphique à l'appui, l'ensemble des divers paramètres contribuant au " coût de la transaction " et à sa réussite sont passés en revue pour dessiner le « modèle de la coopération " proposé. Nul doute que la construction européenne saurait en bénéficier - le modèle est exportable -, de même que la reconnaissance sociale et le rôle économique du traducteur dans le monde moderne.

Pour A. Pym, ce statut est lié à une défense de l'interculturel. Le demier des cinq principes a éthiques "qu'il donne en résumé et conclusion de l'ouvrage prône cette forme de coopération. Les deux premiers chapitres du livre en développaient les prémisses, à travers les 
analyses de la figure symbolique des sang-mêlés (Blendlinge) chez Schleiermacher et d'une histoire de hérauts spartiates trouvée chez Plutarque et Hérodote, deux beaux exemples de lecture herméneutique même si nous n'en partageons pas les interprétations, notamment sur les questions de l'altérité et de la responsabilité, éthiques par excellence au sens philosophique, et si nous résistons à la présentation de Schleiermacher comme adversaire de l'interculturel (pp. $24 s q$.). Dans les deux cas, sont valorisés la position d'intermédiaires, " l'espace de l'entredeux " et des " cultures régionales ", des " Zwischenstaaten ", qu'une idéologie monolithique de la traduction, forte d'une appartenance nationale et uniculturelle, tend à occulter. Intermédiaires réels, humains, communautés géo-historiques qu'il ne suffit pas de poser idéalement : "C'est la raison pour laquelle nous mettons en question tous les modèles où l'interculturalité est purement textuelle, où elle ne s'attache aux traductions que dans le cadre d'une seule langue ou culture et où elle presuppose, de ce fait, les limites de cette langue et de cette culture. " (p. 39) Si nous ne suivons pas l'auteur quand il associe cette attitude à la théorie littéraliste en traduction, nous partageons sa condamnation des pensées de la séparation et de la frontière. " Devenir traducteur, c'est d'abord voyager. " (p. 63) Et il précise aussitôt pour dissiper tout risque d'essentialisme : * C'est seulement ensuite qu'on atteint la condition de Blendling. [...] Est-ce qu'on naît traducteur? Est-ce que le traducteur est natif ou naturel? C'est plutôt en voyageant vers d'autres cultures, en s'engageant dans les déplacements culturels, qu'on devient traducteur, genre particulier de Blendling, genre particulier de héraut."

Au nom de cette proximité de vues, qu'on nous permette d'encore discuter un choix lexical de l'auteur. Car l'interculturel, pas plus que le bilinguisme pour reprendre un exemple classique, n'est garant d'une quelconque faculté traductrice ni de son éventuelle efficacité. L'interculturel $\mathrm{n}$ 'est pas encore le traductif; celui-ci commence avec le transculturel, le cheminement vers l'altérité et sa rencontre, le va-et-vient, la contamination réciproque des cultures, le métissage (voir F. Laplantine et A. Nouss, Le métissage, Flammarion, Dominos 1997). Ce qu'adéquatement nous apprennent les spectres sémantiques des termes traduction et translation. Mais sur ce point, une précision autobiographique d'A. Pym, d'origine australienne, enseignant en Espagne, nous conforte : * C'est souvent un traducteur qui parle [...]. Et c'est aussi, bien évidemment, un étranger qui, refusant d'être traduit, se 
sert d'une langue qui n'est pas tout à fait la sienne. Par là-même, l'interculturalité devient plus qu'une simple hypothèse de travail; elle fait état de ses racines dans l'expérience vécue [...]. » (p. 17) Au-delà de la réussite indéniable de l'entreprise, saluons là un geste proprement éthique.

Alexis Nouss Université de Montréal 